\title{
Community Cloud Architecture to Improve Use Accessibility with Security Compliance in Health Big Data Applications
}

\author{
Samaikya Valluripally, Murugesan Raju, Prasad Calyam, \\ Matthew Chisholm, Sai Swathi Sivarathri, Abu Mosa, Trupti Joshi \\ University of Missouri-Columbia, USA \\ svbqb@mail.missouri.edu,rajum@mail.missouri.edu,calyamp@missouri.edu,mrcd67@mail.missouri.edu,sss26x@mail. \\ missouri.edu,mosaa@health.missouri.edu,joshitr@health.missouri.edu
}

\begin{abstract}
The adoption of big data analytics in healthcare applications is overwhelming not only because of the huge volume of data being analyzed, but also because of the heterogeneity and sensitivity of the data. Effective and efficient analysis and visualization of secure patient health records are needed to e.g., find new trends in disease management, determining risk factors for diseases, and personalized medicine. In this paper, we propose a novel community cloud architecture to help clinicians and researchers to have easy/increased accessibility to data sets from multiple sources, while also ensuring security compliance of data providers is not compromised. Our cloud-based system design configuration with cloudlet principles ensures application performance has high-speed processing, and data analytics is sufficiently scalable while adhering to security standards (e.g., HIPAA, NIST). Through a case study, we show how our community cloud architecture can be implemented along with best practices in an ophthalmology case study which includes health big data (i.e., Health Facts database, I2B2, Millennium) hosted in a campus cloud infrastructure featuring virtual desktop thin-clients and relevant Data Classification Levels in storage.
\end{abstract}

\section{KEYWORDS}

Smart Healthcare, Cloud Architecture, Big Data Application, Security Standard Compliance, Electronic Health Records

\section{ACM Reference format:}

Samaikya Valluripally, Murugesan Raju, Prasad Calyam, Matthew Chisholm, Sai Swathi Sivarathri, Abu Mosa, Trupti Joshi. 2019. Community Cloud Architecture to Improve Use Accessibility with Security Compliance in Health Big Data Applications. In Proceedings of International Conference on Distributed Computing and Networking, Bangalore, India, fanuary 4-7, 2019 (ICDCN '19), 4 pages.

https://doi.org/10.1145/3288599.3295594

\section{INTRODUCTION}

Health Care Systems collect big data using many entities such as instruments, billing records over several years. Increasingly, health care enterprises (both large organizations as well as individual providers) are taking advantage of state-of-the-art technologies in order to extract meaningful insights out of their big data. The

Permission to make digital or hard copies of all or part of this work for personal or classroom use is granted without fee provided that copies are not made or distributed for profit or commercial advantage and that copies bear this notice and the full citation on the first page. Copyrights for components of this work owned by others than ACM must be honored. Abstracting with credit is permitted. To copy otherwise, or republish, to post on servers or to redistribute to lists, requires prior specific permission and/or a fee. Request permissions from permissions@acm.org.

ICDCN '19, fanuary 4-7, 2019, Bangalore, India

(C) 2019 Association for Computing Machinery.

ACM ISBN 978-1-4503-6094-4/19/01 .\$15.00

https://doi.org/10.1145/3288599.3295594

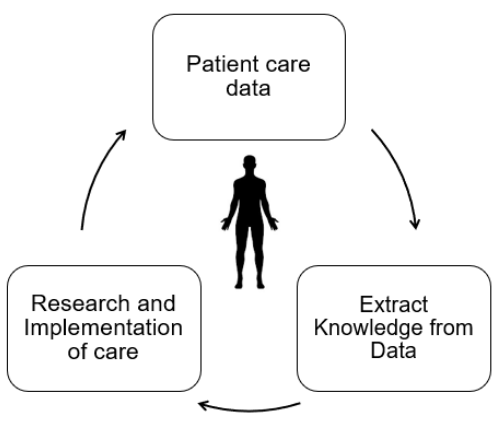

Figure 1: Big data analytics integration enables precision medicine within a Continuous Learning Healthcare System cycle.

goal in such data-driven health care eco-systems is to bridge the knowledge gaps in the information management between patients, health care providers and medical researchers as shown in Figure 1. The expectation is to leverage large health care related data sets to find new risk factors related to a disease, drive clinical research and ultimately inform clinical decisions for smart healthcare.

For successful adoption of big data analytics in healthcare applications as shown in Figure 1, several challenges around data sets need to be simultaneously addressed such as: compliance with stringent security standards, handling of the growing size/diversity of health care data, privileged access to clinical data or proprietary analytic tools, and high-speed processing of data sets with scalability considerations [1]. In addition to the above broad challenges, specific issues that are related to data exchange and integration across the multiple domains include e.g., the lack of homogenization initiatives to handle: (a) multi-source data from clinicians, EHRs and Researchers, (b) diverse file formats with different data classification levels, and (c) security policy management for compliance with inter-organization security standards in the lifecycle stages i.e., billing (PCI DSS standard [2]), data access (HIPAA (Health Insurance Portability and Accountability Act) standard [3]), cyberinfrastructure configuration (NIST standard [4]). As a result, a health big data application owner today has the burden to evaluate computing, networking as well as storage options to implement existing security standards, identifying gaps in achieving scalable application performance, while having the flexibility to test bold hypotheses relevant to e.g., clinical care and drug discovery.

In this paper, we address the above challenges by proposing a community cloud architecture that helps end users (clinicians, researchers, data scientists) improve the use accessibility of health big data across the life cycle stages, while also supporting middleware frameworks that broker the multi-domain resources and align them with security standards compliance. Our main contributions in this work include: 
- Threat modeling of a health big data application workflow

- Security Compliance with standards e.g., HIPAA, NIST

- Novel community cloud architecture supporting data transformation via a secure gateway and thin-client user access supported via a virtual desktop cloud (VDC) [5] [6]

The remainder of the paper is organized as follows: Section 2 presents the related work. Section 3 presents our problem formulation for the threat model and the health big data application performance/security requirements via a case study. Section 4 details our proposed community cloud architecture. Section 5 concludes the paper with a summary of our community cloud architecture implementation.

\section{RELATED WORK}

A data acquisition and analysis framework that captures, stores, correlates and coordinates real-time digital data in a trusted manner before the data is shared or widely accessed is proposed in [7]. In our work, we use a similar framework to store, process, analyze and distribute patient health data in a scalable manner or even in real-time within secure environment for data access. Authors in [7] propose a framework which is efficient in dealing with highvolume and fast-changing workload of heterogeneous types of data processing. Our work is alike in terms of dealing with diverse data sets at large scale. Our architecture addresses problems such as mismatched performance due to data access policies that improve security but introduce bottlenecks through stringent firewall rules and low responsive queries to protect against external threats.

Industry efforts such as in [8] aim at proposing variants of community cloud architectures to deliver health care services in precision medicine with minimal cost, high clinical value, and high usability. Our proposed solution also features high-speed computational capabilities in a campus computing environment, and outlines a novel HIPAA aligned compliance module within a multi-domain resource brokering framework to serve the needs of the end users in health care big data applications. Existing works [9] and [10] pertaining to security and dependability for community-cloud resources in data-intensive research communities mostly deal with security measures and point solutions to counter confidentiality, availability and integrity threats. They also do not consider end-toend security design that helps in dynamic allocation and adaptation using such measures. Our method in this work for performance alignment with security standards is extended from our prior work in [11] that catered to bioinformatics application use cases. Health big data communities can benefit from our proposed solution approach of resource allocation based on multi-domain security requirements, and can augment their current approach of manual co-ordination of policies to achieve end-to-end security alignment.

\section{HEALTH APPLICATION CASE STUDY}

\subsection{Health Facts based Study Motivation}

In this section, we present a big data health application system that motivates our proposed architecture for an integrated high performance scalable solution. The Health Facts database was used in our prior study [12] to investigate the risk factors for age related ophthalmological illness such as cataracts. An electronic medical record system is used in the Health Facts case study, which captures clinical events from hospital procedures, medication history, demographics, encounter, diagnosis, laboratory test, hospital information and billings. Over 600 individual sites from 90 health

\begin{tabular}{|l|l|}
\hline FACT Tables & Number of Records \\
\hline HF_F_clinical_event & $5,606,006,254$ \\
\hline Hf_f_diagnosis & $816,622,927$ \\
\hline Hf_f_encounter & $461,206,375$ \\
\hline Hf_f_enc_history & $309,634,504$ \\
\hline Hf_f_implant_log & 33,567 \\
\hline Hf_f_lab_procedure & $4,293,382,357$ \\
\hline Hf_f_medication & $634,846,774$ \\
\hline Hf_f_med_history & $208,259,928$ \\
\hline Hf_f_microbiology & $156,748,165$ \\
\hline Hf_f_micro_susceptibility & $112,372,182$ \\
\hline Hf_f_procedure & $112,153,607$ \\
\hline Hf_f_surgical_case & $5,047,689$ \\
\hline Hf_f_surgical_procedure & 396,476 \\
\hline
\end{tabular}

Table 1: High scale of records involving FACT tables accessed within a big data health application.

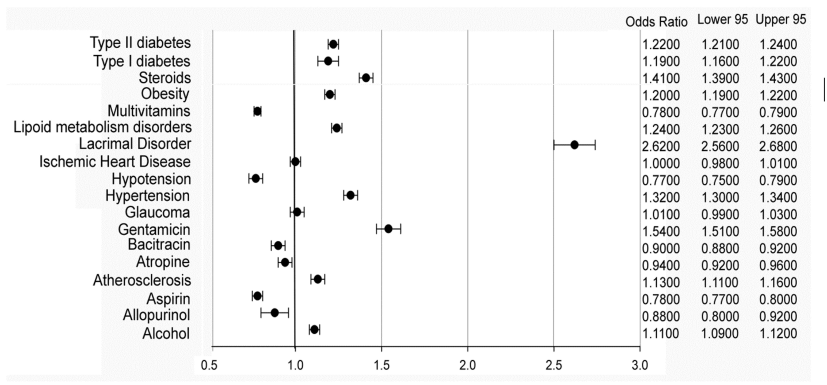

Figure 2: Risk analysis that highlights the nine risk factors pertaining to an ophthalmology study featuring a health big data application [12].

systems were participating in contributing data to the Health Facts database with billions of records as shown in Table 1. Using the Health Facts database, the results obtained from statistical analysis [12] are shown in Figure 2. They pertain to nine risk factors with over a $20 \%$ increase in odds of developing the ophthalmological illness such as a cataract. The statistical analysis looked at 18 different variables to determine their effect on cataract formation. Odds ratio analysis showed that 7 out 18 variables had a $20 \%$ or higher odds of a cataract diagnosis. It was determined that 3 factors had lower than $20 \%$ odds of cataract association. With such an insight, researchers can have more informed methods in developing novel treatments for ophthalmological illness cases. In order to have an effective big data analytics eco-system, the Health Facts database has to cater to a health big data application's performance and security requirements listed in the following sub-sections with an appropriate co-design of system considerations.

\subsection{Performance Requirements}

During the studies in [12] for determining the risk factors in health big data, significant performance hindrances were discovered that led to slow query responses. The usability was affected due to the inaccessibility of the data specifically to run large-scale queries for analytical purposes. The end users who are typically not experts in high-performance computing require automation for handling their queries that involve performing a data lookup at the high scale shown in Table 1 . It took approximately 24 minutes to run a basic program using about $1 \%$ of the data available in the Health Facts database. For more complex programs using more of the data, it took 3 or 4 days to run. Such a performance output is prohibitively slow for researchers or clinicians who would need to wait excessively to test bold data-driven hypotheses and derive analytical insights for the future illness treatment or drug discovery. Such inefficient performance output in workflows can lead to wastage of valuable 
researcher time and cost, while also slowing the pace of innovation. In addition to such practical problems, additional bottlenecks in utilizing large health related databases can arise due to slow disk mechanisms used for data lookup, inadequate memory provisioning, and low-scale processing backends. Particularly when there are billions of records to be scanned within query look up in the tables, relevant user interfaces and appropriate system configurations need to be designed to obtain times for retrieval of relevant records in an efficient and effective manner for the users.

\subsection{Security Requirements}

The current security policies followed by major health data providers are a combination of policies given by EHR Vendors, IRB at local institutions [13] compliant with HIPAA, and server warehouse limitations within a campus environment. Each of the multi-source data (e.g., Health Facts, imaging data from scientific instruments, other healthcare vendors source data) are typically available in different formats and the researchers have the burden to ensure compliance in their studies across all these different source data with different standards. There is a need to homogenize these policies with a standard format that is compliant with NIST guidelines [4] for data classification levels as the data traverses across various domains with stringent policies [11]. Bearing in mind the challenges for compliance with diverse data sets with heterogeneous standards involving EHR Vendors, institutional policies and user requirements, it is essential to have end-to-end security alignment with a secure gateway for data access.

\section{COMMUNITY CLOUD SOLUTION DESIGN}

\subsection{Threat model}

The community cloud design has to consider different types of threats involved in the life cycle stages of a health big data application such as the one in our case study with the Health Facts database. For the threat modeling, we adapt the Microsoft's STRIDE model [14]. In our investigation of the case study requirements, we have noted that a more common concern for users at the data-level relates to the data access levels (i.e., Loss of Confidentiality) for data-in-motion and data-at-rest and data-in-use within the lifecycle stages. There is concern of unintended users having access to data due to provisioning of excessive privileges amongst the various roles. This is particularly important with healthcare data as it is typically confidential data that must be kept private. Loss of Integrity, where data may be corrupted due to administrator error in handling databases, or if a user action causes disk space to exceed for an analysis process. This is a major concern for those who are analyzing the data. Lastly, there is a threat of users not being able to access their data when needed (i.e., Loss of Availability) due to e.g., administrator error involving inadvertent system management actions which cause major changes to storage that may not get notified to users in a timely manner, which may then result in partial or full loss of their data.

\subsection{Security Alignment and Compliance}

One of the major hurdles for performance and security co-design driven resource management is that in most of the cases the domain security requirements are diverse. Thus, we need to homogenize the different data forms and align the security and performance requirements for compliance. However, the data classification levels (DCL) for multi-domain resources are of different levels within enterprises (DCL3,4) that are relatively difficult to align such disparity in DCL. To address this issue, we adapt a novel alignment scheme detailed in [11] to homogenize such diverse security requirements into homogeneous policy statements. As the Health Facts database comprises of sensitive healthcare data from day-today patient encounters, there is a need to utilize the dataset in a manner that ensures it is consistent with applicable regulations and has appropriate compliance levels when users are trying to access and analyze data. The policies and procedures for utilization of Health Facts database is discussed in Health Facts Access and Data governance [15].

SOM-R-007: Users will access Health Facts through a secure server that is DCL3 compliant is a Health Facts policy [15] which is about data governance that is compliant with HIPAA. The alignment results in a homogenization of different data formats into a unified format that is HIPAA compliant and can be used with highperformance in a community cloud architecture within a URB as shown in Figure 3. To check the compliance between the resource and security specifications, we consider the rules mentioned in NIST SP800-53 [4] where different security controls are classified as Low, Medium, and High categories. Now with the mapping document between NIST and HIPAA rules, we can apply similar categorical levels within our Health Facts based health big data application case study involving ophthalmological illness.

\subsection{Architecture Components}

Our proposed community cloud architecture shown in Figure 3 involves various components such as Source Data, Data Validation Engine, Secure and Trusted gateway, URB, VDC with easy-to-use health big data application User Interface (UI). Data validation is performed for effective performance before uploading external data into the community cloud for a user study purpose. To ensure that existing security rules implemented in the Health Facts application are not violated, we make sure the Personal Identified information is not taken into the cloud platform as the researchers require mostly diagnosis information. Any sensitive data personal information, Medical Record Number (MRN) that is tagged along with the extracted information by the researchers for the analysis, will be anonymized and assigned with a new temporary value for each new session the researcher tries to access the database. Our cloud architecture is designed in a way where it doesn't store/cache any sensitive data to avoid any attacks. Therefore, the data is deleted from cloud after every remote desktop session is ended. In addition to this, as shown in Figure 3 a federated Identity Access Management (IAM) is used to authenticate a user who tries to access the analytical data with appropriate authorized privileges.

Considering the multi-format in this input data, our proposed community cloud architecture homogenizes the data classification levels and ensures all the alignment is of HIPAA compliance. After checking the compliance of the data with Health Facts policies (HIPAA compliant), to ensure secure transfer of data throughout the system, a secure gateway is used. This secure gateway has the implementation of appropriate security controls to enable the users to run the analysis on the queried data using relevant computation tools. To ensure high performance for the users at the VDC's as shown in the Figure 3, our approach requires a query parallelization mechanism in the community cloud. The parallel querying module enables the users to run more than a query on the data pulled into the community cloud by the researchers. In the current Health Facts application case study, parallel queries are executed as different 


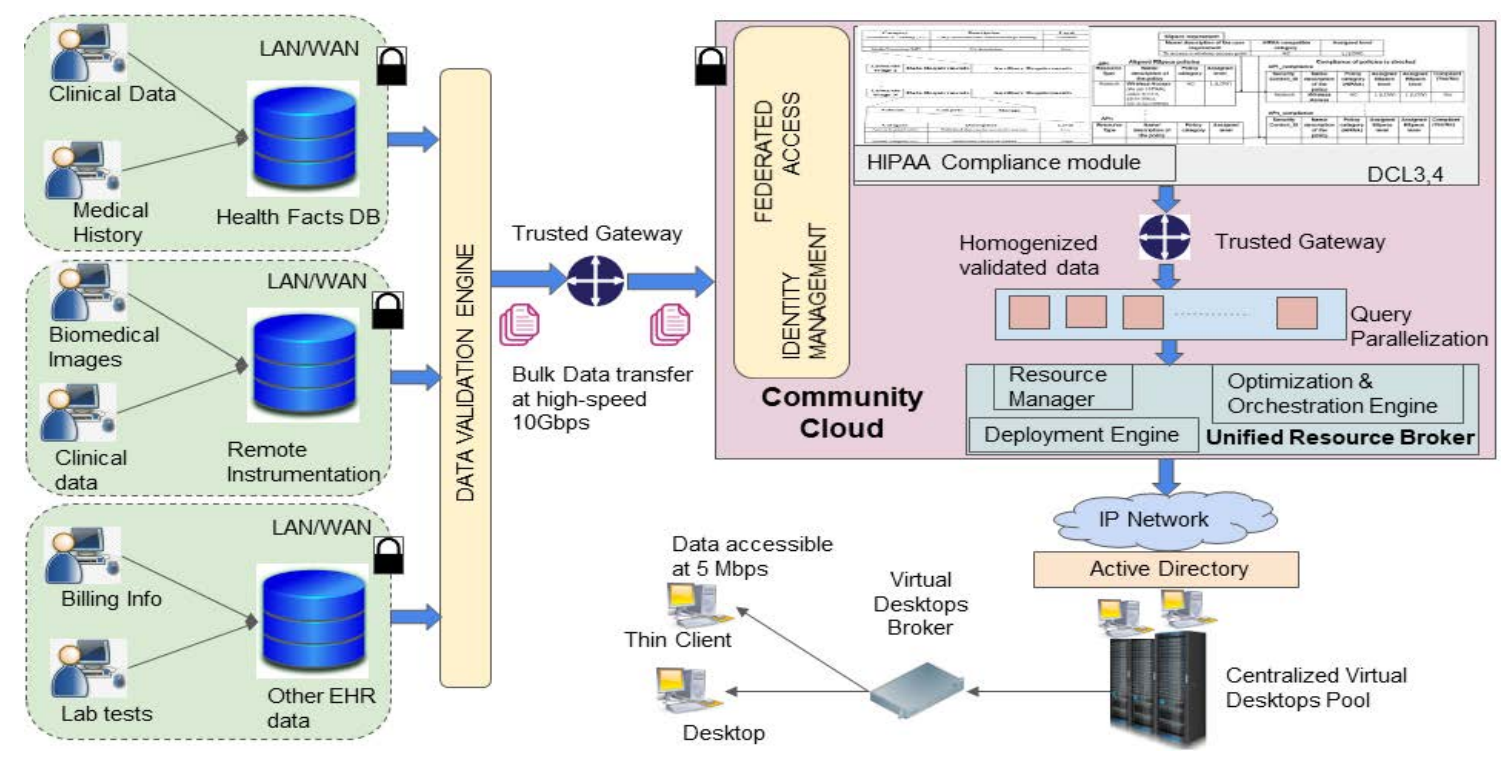

Figure 3: Integrated community cloud design with security compliance of heterogeneous data formats using a unified brokering structure to enhance the performance of querying data for analysis of a big data health application

SAS sessions with equal share of resources to compute. However, this will lead to a bottleneck in performance as there might be a few queries which require less resources than the equal share currently being implemented in Health Facts application. Thus, our proposed architecture implements a prioritized queue for the queries to run using an URB as shown in Figure 3. The functionality of this URB is used to manage the resource needs of a user, by ensuring that the required amount of resources are available to service the users in order to fulfill their analytics objectives. At the end-user side, data could be accessed by clinicians/researchers only through VDC which will be deleted for every new session to avoid any risk of unauthorized sensitive data downloads.

\section{CONCLUSION}

Smart healthcare with big data analytics has great potential to foster medical advancements in areas such as precision medicine, determination of risk factors, and biomedical research. In this paper, we proposed a novel community cloud architecture to automate any existing semi-automated big data health application that uses large healthcare related databases to test bold hypotheses. Our approach involves a co-design of high-scale performance and security compliance through alignment of user requirements and data provider policies via a URB module. Using a case study involving a ophthalmological illness data analysis use case with multiple data sources, we describe how our community cloud architecture can mitigate the query response latency in running large-scale queries over billion transaction records, while also ensuring compliance with heterogeneity in the data classification levels in the various lifecycle stages of the health big data application.

\section{ACKNOWLEDGMENTS}

This work was supported in part by the National Science Foundation under award number OAC-1827177. Any opinions, findings, and conclusions or recommendations expressed in this publication are those of the author(s) and do not necessarily reflect the views of the National Science Foundation.

\section{REFERENCES}

[1] J. Tenenbaum, P. Avillach, M. Benham-Hutchins, M. Breitenstein, E. Crowgey, M. Hoffman, X. Jiang, S. Madhavan, J. Mattison, R. Nagarajan, B. Ray, D. Shin, S. Visweswaran, Z. Zhao and R. Freimuth, "An informatics research agenda to support precision medicine: seven key areas", Journal of the American Medical Informatics Association, 2016.

[2] PCIDSS standard. [Online]. Available at: https://www.pcisecuritystandards.org/pci security [Accessed 10-15-2018].

[3] "An Introductory Resource Guide for Implementing the Health Insurance Portability and Accountability Act (HIPAA) Security Rule", NIST Special Publication 800-66 Revision 1, 2008.

[4] "Security and Privacy Controls for Federal Information Systems and Organizations", NIST SP800-30 Technical Report, 2013.

[5] P. Calyam, S. Rajagopalan, S. Seetharam, A. Selvadhurai, K. Salah, R. Ramnath, "VDC-Analyst: Design and Verification of Virtual Desktop Cloud Resource Allocations", Elsevier Computer Networks fournal (COMNET), 2014

[6] P. Calyam, S. Rajagopalan, A. Selvadhurai, S. Mohan, A. Venkataraman, A. Berryman, R. Ramnath, "Leveraging OpenFlow for Resource Placement of Virtual Desktop Cloud Applications", IFIP/IEEE Intl. Symposium on Integrated Network Management (IM), 2013

[7] P. Nguyen, S. Konstanty, T. Nicholson, T. Brien, A. Schwartz-Duval, T. Spila, K. Nahrstedt, R. Campbell, I. Gupta, M. Chan, K. McHenry, N. Paquin, "4CeeD: Real-Time Data Acquisition and Analysis Framework for Material-related CyberPhysical Environments", 17th IEEE/ACM International Symposium on Cluster, Cloud and Grid Computing (CCGRID), 2017.

[8] Google cloud for Healthcare: new APIs, customers, partners and security updates. [Online]. Available at:https://www.blog.google/products/google-cloud/googlecloud-healthcare-new-apis-customers-partners-and-security-updates/ [Accessed 10-15-2018].

[9] C.L. Chen, C. Zhang, "Data-intensive applications, challenges, techniques and technologies: A survey on Big Data", Information Sciences, 2014.

[10] H. Takabi, J. Joshi, G. Ahn, "Security and Privacy challenges in cloud computing environments", IEEE Security and Privacy fournal, Vol. 8, No. 6, pp. 24-31, 2018.

[11] M. Dickinson, S. Debroy, P. Calyam, S. Valluripally, Y. Zhang, R.B. Antequara, T. Joshi, T. White, D. Xu, "Multi-cloud Performance and Security Driven Federated Workflow Management", IEEE Transactions in Cloud Computing, 2018.

[12] M. Raju, M. Chisholm, A. Mosa, Greg Petroski, Chi-Ren Shyu, and Frederick W. Fraunfelder, "Investigating Risk Factors for Age-Related Cataract Using the Cerner Health Facts Database", Fournal of Eye and cataract surgery, 2017.

[13] Institutional Review Board at University of Missouri. [Online]. Available at: https://research.missouri.edu/irb/ [Accessed 10-11-2018].

[14] Microsoft threat modeling tool preview and template documentation. [Online]. Available at:https://docs.microsoft.com/en-us/azure/security/azure-securitythreat-modeling-tool-getting-started [Accessed 10-15-2018].

[15] "Health Facts access and data governance", [online]. [Accessed 10-11-2018]. 